\title{
The prevention of early-onset group B streptococcal infections in the newborn
}

$\mathrm{G}$ ROUP B STREPTOCOCCI (GBS) ARE A MAJOR CAUSE OF morbidity and mortality among newborn infants. Various strategies to prevent infection in the newborn were reviewed by the Infectious Diseases and Immunization Committee and the Fetus and Newborn Committee of the Canadian Paediatric Society and by the Maternal/Fetal Medicine Committee of the Society of Obstetricians and Gynaecologists of Canada. The committees identified an urgent need for additional re-

This Consensus Statement is being concurrently published in the Canadian Journal of Paediatrics and the Journal of the Society of Obstetricians and Gynaecologists of Canada

Correspondence: Infectious Diseases and Immunization Committee, Canadian Paediatric Society, 401 Smyth Road, Ottawa, Ontario K1H 8L1. Telephone (613) 737-2728, Fax (613) 737-2794 search to provide Canadian data upon which to base the selection of the optimal strategy. Until the results of these studies are available, this National Consensus Statement provides guidance for identification and management of women whose newborns may be at risk of group B streptococcal (GBS) disease.

\section{BACKGROUND}

GBS (Streptococcus agalactiae) continue to be a major cause of bacterial sepsis among newborns. The source of infection in the neonate is the colonized maternal birth canal; transmission occurs before or during the birth process. Estimates of GBS colonization rates among pregnant women range from 15 to $40 \%$ (1-4). GBS is transmitted to 40 to $70 \%$ of newborns of colonized mothers; however, only 1 to $2 \%$ of such infants develop 
disease (5). Two types of GBS infections occur in the newborn. Early-onset disease (less than seven days of age) is more common and has a higher rate of mortality, and it is the subject of this statement. Late-onset disease (seven days to three months of age) is less common and has a lower associated mortality (6-8).

Factors that have been associated with increased risk of early-onset GBS disease include: maternal age less than 20 years; low socioeconomic status; gestation less than 37 weeks; prolonged rupture of membranes (greater than $12 \mathrm{~h}$ ); maternal intrapartum pyrexia (temperature greater than $37.5^{\circ} \mathrm{C}$ ); multiple pregnancy; GBS bacteriuria; and degree of maternal genital colonization (heavy versus light) $(5,9,10)$. Colonization rates are relatively constant during pregnancy. Fewer than 10\% of women who are culture-negative late in the second trimester are culture-positive at delivery $(1,2)$.

\section{INTERVENTION STRATEGIES}

The high rates of morbidity and mortality associated with GBS infections and the rapidity of onset of postnatal disease have led to numerous strategies to prevent infection in the newborn. They include antenatal, intrapartum and postnatal chemoprophylaxis, and active and passive immunoprophylaxis. Controversy persists about the optimal strategy because of insufficient data or differing interpretations of existing data.

Immunoprophylaxis - Active: Serum antibody to GBS capsular polysaccharide correlates with protection against infection. Antibody levels to GBS in infected infants and their mothers are lower than in uninfected maternal-newborn pairs (11). This has led to efforts to develop GBS capsular polysaccharide vaccines for the active immunization of pregnant women to prevent early-onset and late-onset disease in the newborn (12). As with other bacterial vaccines, a substantial number of individuals do not respond to polysaccharide antigens either because of genetic influences such as immunoglobulin allotype or because of the T cell-independent nature of the antigen (13). Lack of significant placental transfer of immunoglobulin (Ig) G before 32 weeks of gestation also diminishes the usefulness of this intervention for protection of the very premature infant. However, efforts to develop an immunogenic vaccine that can be administered to all women, or during pregnancy, continue to be made. Although active immunization may be the ultimate solution, no vaccine is likely to be available in the foreseeable future.

Passive: The use of intravenous immunoglobulin (Ig-IV) has been proposed to increase antibody titres to GBS in newborns. Clinical studies using passive immunoprophylaxis have been disappointing (14). Variability in GBS-specific antibody in Ig-IV preparations may account for these poor results $(15,16)$. Although recent efforts to produce a GBS hyperimmune Ig-IV preparation may make passive immunization a viable intervention in the future (17), at present, routine use of Ig-IV cannot be recommended for prevention or therapy of GBS infection in newborns (18).

Chemoprophylaxis: Several strategies have been employed using antibiotics for the prevention of GBS infections in the newborn. These can be categorized as neonatal, antepartum and intrapartum. However, no strategies prevent all cases of early-onset GBS disease. Fulminant GBS sepsis and neonatal demise can occur in spite of any planned intervention.

Neonatal: Although penicillin or ampicillin therapy of the neonate may prevent some cases of GBS in term infants (19), more than $60 \%$ of infants are already symptomatic at, or shortly after, birth and are at high risk of GBS complications and poor outcome $(7,9,20,21)$. Chemoprophylaxis of neonates appears to be an inferior strategy for prevention of GBS infections.

Antepartum: The efficacy of antibiotic therapy of GBScolonized women during pregnancy for preventing neonatal infection has not been established. Studies using newborn infection as an outcome measure have been of insufficient size to demonstrate efficacy (22-24). Studies in which maternal GBS colonization was followed after a course of antibiotic therapy have demonstrated high rates of GBS recurrence (67\%) by the time of delivery. This was true even when antibiotics were given to both pregnant women and their sexual partners $(7,25)$. An exception to the lack of benefit of antepartum antibiotics is women with GBS bacteriuria, which increases the risk for premature rupture of membranes and preterm labour. Oral antibiotic therapy resolves GBS bacteriuria and decreases the risk of preterm labour, although genital colonization typically persists $(2,10)$.

Intrapartum: Most experts agree that intrapartum maternal chemoprophylaxis is the best strategy available to prevent early-onset neonatal GBS disease $(7,8,21,26)$. Several studies have assessed the benefit of intrapartum chemoprophylaxis (3,27-31); a meta-analysis of selected randomized controlled trials $(5,29-31)$ showed benefit of intrapartum prophylaxis (32). The strategies used by the studies differ primarily in the methods used to select women for prophylaxis (including whether and when to screen pregnant women for colonization with GBS), laboratory methods to detect GBS colonization, and selective or nonselective chemoprophylaxis.

Timing of GBS screening: Anorectal and vaginal swabbing for GBS culture early in the third trimester (26 to 28 weeks) permits adequate time to identify GBS colonization. Problems with this approach include late acquisition of colonization and lack of prenatal care. A single culture has a predictive value of GBS colonization at delivery of only $67 \%$ (27). Screening late in the third trimester (36 weeks) decreases the likelihood of missing late acquisition of GBS but misses women with premature labour, and therefore, neonates who are at highest risk of GBS complications. A strategy that screens women presenting in premature labour (less than 37 weeks) and that treats GBS carriers or those 
TABLE 1

Research needs for Canada

- GBS colonization rates in Canadian pregnant women

- GBS disease rates and risk factors in newborn infants

- Cost and availability of the best laboratory test for maternal GBS colonization

- Outcome of newborns after various intrapartum chemoprophylaxis strategies

- Cost analysis of various prevention strategies

- Pilot demonstration and feasibility projects to assess proposed strategies

- Investigation of GBS vaccines and other primary preventative strategies

GBS Group B streptococcus

women in whom the test result is unavailable would result in the prophylaxis of as many as $7.5 \%$ of pregnant women (33), but it does not address term infants, who account for over two-thirds of early-onset GBS disease. Intrapartum screening suffers from the lack of rapid, sensitive laboratory tests, which would provide the opportunity to make decisions about chemoprophylaxis with sufficient time before delivery.

Laboratory tests: Chemoprophylaxis strategies that involve identification of GBS-colonized women require tests with high sensitivity and specificity. The rate of isolation of GBS is improved through culture of the lower vagina and anorectum and through use of selective media for transport and culture $(1,7)$. Bacterial isolation is time-consuming, and results are not rapidly available for the woman presenting in labour. Optimal use of chemoprophylaxis requires a sufficient interval (at least 4 h) between beginning antibiotics and delivery (34). Antigen detection tests provide results more quickly (5 h or less) but with loss of sensitivity relative to culture (29,35-39). The shortcomings of the laboratory tests have led to strategies for selective chemoprophylaxis based only on epidemiological risk factors without laboratory sampling $(27,40)$.

Selective versus nonselective prophylaxis: Identification of risk factors that increase the likelihood of GBS infection in the newborn of a colonized woman has led to strategies for use of prophylaxis in high risk situations such as preterm labour (less than 37 weeks), prolonged rupture of membranes (greater than $12 \mathrm{~h}$ ) and intrapartum maternal fever. This strategy has been shown to be efficacious $(21,41,42)$ but is estimated to fail to prevent 25 to $30 \%$ of cases of early-onset GBS infection $(9,21)$. Reasons for this failure are: infection in infants of mothers with rupture of membranes at less than $12 \mathrm{~h}$; lack of maternal fever; and women receiving late prenatal care in whom GBS colonization is not recognized. Treatment based on risk factors alone without prior GBS screening has been reported in one selected population (9) but has similar limitations in prevention of neonatal GBS infection. Prophylaxis of all colonized women regardless of risk factors would de-
TABLE 2

Risk factors for which intrapartum chemoprophylaxis is recommended

1. Preterm labour ( $<37$ weeks' gestation)

2. Term labour ( $\geq 37$ weeks' gestation)

a. Prolonged rupture of membranes: chemoprophylaxis should be given if labour is likely to continue beyond $18 \mathrm{~h}$ (neonatal benefits are optimally achieved if antibiotics are given at least $4 \mathrm{~h}$ before delivery) b. Maternal fever during labour $\left(>38^{\circ} \mathrm{C}\right.$ orally)

3. Previous delivery of a newborn with GBS disease regardless of current GBS colonization status

4. Previously documented GBS bacteriuria

GBS Group B streptococcus

crease the number of undetected cases (except for the late prenatal care) but would dramatically increase the use of intrapartum antibiotics, with the associated risks of adverse reactions and, depending on the colonization rate of the population, possibly the loss of cost-effectiveness. Although identification of women with heavy GBS colonization predicted a higher risk of newborn GBS infection, $16 \%$ of infections occurred in newborns of lightly colonized women (28), suggesting that this stratification method was of limited benefit. More simply, increased sensitivity might be achieved by expanding the list of risk factors that would be indications for chemoprophylaxis (ie, GBs bacteriuria, multiple births in preterm labour).

Cost-effectiveness: Several strategies have been shown to be cost-effective in the United States, including screening at 26 to 28 weeks and selective intrapartum chemoprophylaxis (43), intrapartum screening and chemoprophylaxis of all GBS-colonized women regardless of risk factors (44) and intrapartum prophylaxis based on risk factors only (43). The clinical outcomes and costs of 19 potential strategies were recently compared using decision analysis (45). Universal intrapartum chemoprophylaxis, intrapartum chemoprophylaxis based on risk factors, and universal screening at 36 weeks' gestation plus intrapartum chemoprophylaxis of all GBS culture-positive women and women in preterm labour were identified as the optimal strategies. However, most of the strategies modelled are theoretical and need to be studied in practice before implementation. None of the cost analyses addressed the substantial implications or costs associated with the investigation and management of neonates whose mothers received intrapartum chemoprophylaxis, especially newborns without GBS disease.

Management of infants of mothers who received intrapartum chemoprophylaxis: No studies have been performed assessing the optimal management of newborns whose mothers received intrapartum chemoprophylaxis; therefore, recommendations are necessarily empirical (46). Management should take into account gestational age and clinical evaluation. 


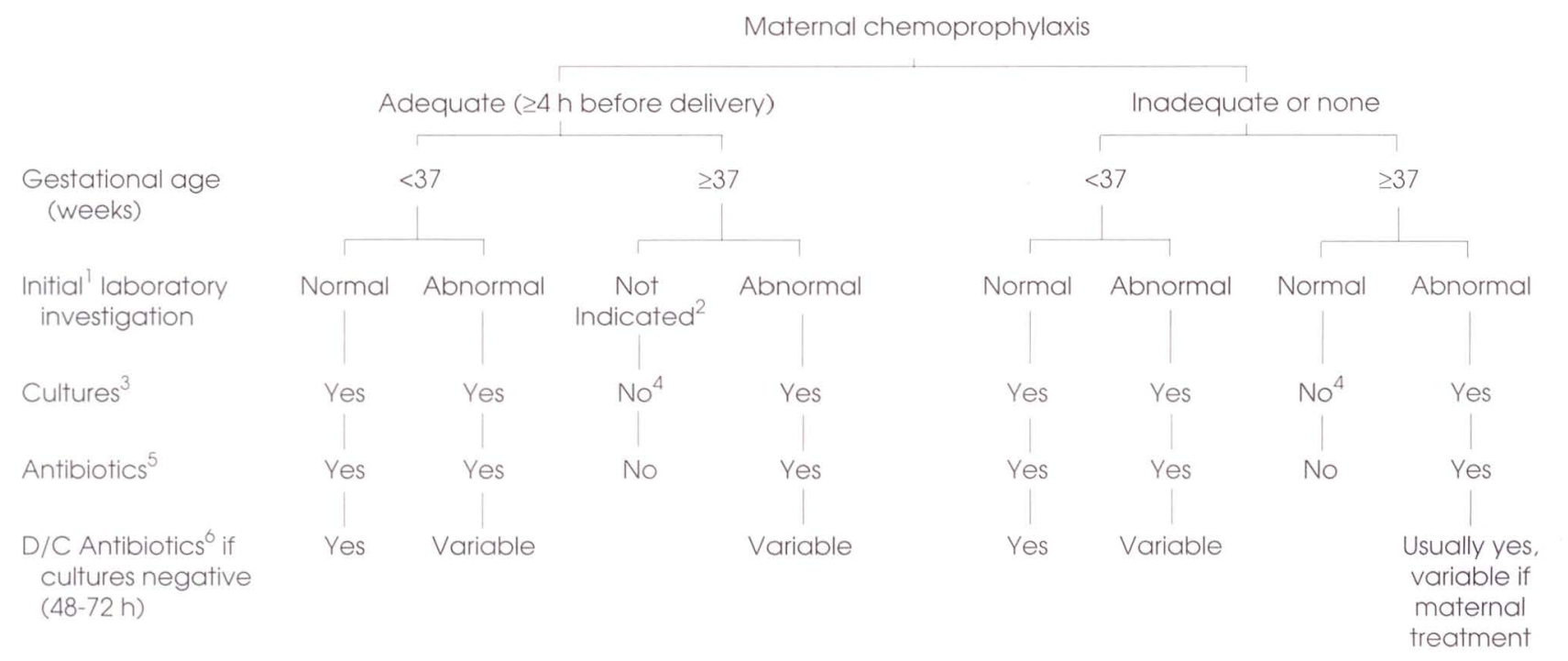

All babies with clinical signs of sepsis should be investigated and treated with antibiotics regardless of maternal GBS colonization or chemoprophylaxis

\footnotetext{
1. Laboratory investigation (not routinely required such as complete blood count with differential, erythrocyte sedimentation rate or C-reactive protein) may assist clinical decisions regarding risk of GBS sepsis.

2. Not necesssarily indicated for all high risk GBS situations; may be clinically indicated with signs of maternal chorioamnionitis.

3. When obtained, a blood culture should be collected in an appropriate resin tube. Pending further guidelines, other cultures, including cerebrospinal fluid (CSF), are at the discretion of the attending physician. Surface cultures and antigen detection (except in the CSF) should not be used to guide therapy.

4. Some clinicians may obtain cultures and treat pending results when there are signs of maternal chorioamnionitis.

5. Antibiotics should always include penicillin or ampicillin. The decision to add another antibiotic (usually an aminoglyloside) is dependent on the assessment of likelihood of infection with bacteria other than GBS.

6. With positive cultures, the baby should receive a full course of therapy. With negative cultures, a variable length of therapy may occur based on the physician's assessment of the likelihood of infection, which may include laboratory abnormalities and potential influence of prior treatment of the mother.
}

Figure 1) Flow chart for infants born with antenatal risk factors for group B streptococcus (GBS) indicating chemoprophylaxis. This paradigm is for use only for babies without clinical signs of sepsis

\section{RECOMMENDATIONS}

Although intrapartum chemoprophylaxis in high risk situations reduces the morbidity and mortality due to early onset of GBS infection, no method prevents all GBS deaths. Detailed review of this information indicates that specific Canadian data are required to determine the best strategy for chemoprophylaxis in the prevention of early-onset neonatal GBS infection (Table 1) and to test various methods of identifying women whose neonates may be at risk of GBS disease. Two widely recommended strategies are universal screening coupled with selective intrapartum chemoprophylaxis of colonized women at high risk or intrapartum chemoprophylaxis of all women at high risk. Currently available data favour the first approach; however, the cost implications of universal screening are considerable.

Until the results of Canadian studies are available, the Infectious Diseases and Immunization Committee and the Fetus and Newborn Committee of the Canadian Paediatric Society, and the Maternal/Fetal Medicine Committee of the Society of Obstetricians and Gynaecologists of Canada recommend the following:
1. The strategy for decreasing early-onset GBS infection in the neonate should be considered an area of urgently needed research in Canada (Table 1).

2. Until more specific information is available, identification and management of women whose newborns may be at increased risk of GBS disease are acceptable by either of two methods:

a. Universal screening of all pregnant women at 26 to 28 weeks' gestation with a single combined vaginal-anorectal swab and selective intrapartum chemoprophylaxis of GBS-colonized women with identified risk factors (Table 2).

b. No universal screening but intrapartum chemoprophylaxis for all women with identified risk factors (Table 2). This strategy should also be used in cases where universal screening is the policy but was either not done or the test results are not available.

3. Where GBS cultures are used, a single swab of the lower vagina and the anorectum should be 
transported to the laboratory in selective broth medium and subcultured onto selective solid media. Standardized methods should be established in each facility for the collection, requisition, transport, testing and reporting of these specimens.

4. Antepartum oral antibiotic therapy should be used for women with GBs bacteriuria. These women should still be considered to be GBS-colonized at the time of going into labour. They do not need to be rescreened once they have been identified.

5. Use of intrapartum chemoprophylaxis of women who previously have given birth to an infant with GBS disease regardless of GBS colonization status.

6. The antibiotic regimen of choice for intrapartum chemoprophylaxis is intravenous ampicillin (2 $\mathrm{g}$ initially followed by 1 to 2 g every 4 to $6 \mathrm{~h}$ ) or penicillin G ( 5 million U every $6 \mathrm{~h}$ ) until delivery or until labour is stopped. Women who are allergic to

\section{REFERENCES}

1. Anthony BF, Eisenstadt R, Carter J, et al. Genital and intestinal carriage of group B streptococci during pregnancy. J Infect Dis 1981:143:761-6.

2. Thomsen AC, Morup L. Hansen KB. Antibiotic elimination of group B streptococci in urine in prevention of preterm labour. Lancet 1987;i:591-3.

3. Allardice JG. Baskett TF, Seshia MMK, et al. Perinatal group B streptococcal colonization and infection. Am J Obstet Gynecol 1982:142:617-20.

4. Regan JA, Klebanoff MA. Nugent RP. Vaginal Infections and Prematurity Study Group. The epidemiology of group $\mathrm{B}$ streptococcal colonization in pregnancy. Obstet Gynecol 1991:77:604-10.

5. Boyer KM, Gadzala CA, Burd LI, et al. Selective intrapartum chemoprophylaxis of neonatal group B streptococcal early-onset disease. 1. Epidemiologic rationale. J Infect Dis 1983;148:795-801.

6. Institute of Medicine. National Academy of Sciences. New Vaccine Development. Establishing Priorities, 1: Diseases of Importance in the United States. Washington: National Academy Press, 1985:424-39.

7. Baker CJ, Edwards MS. Group B streptococcal infections: perinatal impact and prevention methods. Ann NY Acad Sci 1988:549:193-202.

8. Yagupsky P, Menegus MA, Powell KR. The changing spectrum of group B streptococcal disease in infants: an eleven-year experience in a tertiary care hospital. Pediatr Infect Dis J 1991:10:801-8.

9. Schuchat A. Oxtoby M. Cochi S. et al. Population-based risk factors for neonatal group B streptococcal disease: results of a cohort study in Metropolitan Atlanta. J Infect Dis 1990; 162:672-7.

10. McDonald H, Vigneswaran R. O'Loughlin JA. Group B streptococcal colonization and preterm labour. Aust NZ J Obstet Gynaecol 1989;29:291-3.

11. Coleman RT, Sherer DM, Maniscalco WM. Prevention of neonatal group B streptococcal infections: advances in maternal vaccine development. Obstet Gynecol 1992:80:301-9.

12. Baker CJ. Rench MA, Edwards MS, et al. Immunization of penicillin may be given intravenous clindamycin (300 to $600 \mathrm{mg}$ every $8 \mathrm{~h}$ until delivery).

7. Women with a culture positive for GBS at 26 to 28 weeks' gestation do not require treatment unless they become high risk and are in labour.

8. Management of asymptomatic infants of mothers who received intrapartum chemoprophylaxis should be based on the infant's gestational age, results of investigations for sepsis and the adequacy of the maternal intrapartum chemoprophylaxis (Figure 1). Newborns with clinical signs of sepsis should be investigated and treated with antibiotics to cover GBS and other organisms regardless of maternal GBS colonization or chemoprophylaxis. Because of the concern that intrapartum antibiotics could interfere with the ability to obtain a positive blood culture, symptomatic infants in whom blood cultures are negative should receive a full course of antibiotics, the duration of which will depend on the clinical picture.

pregnant women with a polysaccharide vaccine of group B streptococcus. N Engl J Med 1988:319:1180-5.

13. Baker CJ. Rench MA. Kasper DL. Response to type III polysaccharide in women whose infants have had invasive group B streptococcal infection. N Engl J Med 1990:322:1857-60.

14. Fischer GW. Immunoglobulin therapy of neonatal group B streptococcal infections: an overview. Pediatr Infect Dis J 1988:7:S13-6.

15. Baker CJ. Noya FJD. Potential use of intravenous immune globulin for group B streptococcal infection. Rev Infect Dis 1990:12:S476-82.

16. Fischer GW. Immunoglobulin therapy for neonatal sepsis: an overview of animal and clinical studies. J Clin Immunol 1990;10:40S-6S.

17. Fischer GW, Weisman LE. Hemming VG. Directed immune globulin for the prevention or treatment of neonatal group B streptococcal infections: a review. Clin Immunol Immunopathol 1992:62:S92-7.

18. Infectious Diseases and Immunization Committee, Canadian Paediatric Society. Intravenous immunoglobulin use in children. Can Med Assoc J 1992:146:121-4.

19. Siegel JD, McCracken GH Jr. Threlkeld N, et al. Single-dose penicillin prophylaxis of neonatal group $\mathrm{B}$ streptococcal disease: conclusion of a 41-month controlled trial. Lancet 1982:i:1426-30.

20. Pyati SP. Pildes RS, Jacobs NM, et al. Penicillin in infants weighing two kilograms or less with early-onset group B streptococcal disease. N Engl J Med 1983:308:1383-9.

21. Boyer KM, Gotoff SP. Prevention of early-onset neonatal group B streptococcal disease with selective intrapartum chemoprophylaxis. N Engl J Med 1986;314:1665-9.

22. Hall RT, Barnes W, Krishnan L, et al. Antibiotic treatment of parturient women colonized with group B streptococci. Am J Obstet Gynecol 1976:124:630-4.

23. Lewin EB, Amstey MS. Natural history of group B streptococcus colonization and its therapy during pregnancy. Am J Obstet Gynecol 1981:139:512-5.

24. Merenstein GB. Todd WA. Brown G, et al. Group B 
及-hemolytic streptococcus: randomized controlled treatment study at term. Obstet Gynecol 1980;55:315-8.

25. Gardner SE, Yow MD, Leeds LJ, et al. Failure of penicillin to eradicate group B streptococcal colonization in pregnant woman: a couple study. Am J Obstet Gynecol 1979;135:1062-5.

26. American Academy of Pediatrics Committee on Infectious Diseases and Committee on Fetus and Newborn. Guidelines for prevention of group B streptococcal (GBS) infection by chemoprophylaxis. Pediatrics 1992;90:775-8.

27. Boyer KM, Gadzala CA, Kelly PD, et al. Selective intrapartum chemoprophylaxis of neonatal group B streptococcal early-onset disease. II. Predictive value of prenatal cultures. J Infect Dis 1983;148:802-9.

28. Morales WJ, Lim DV. Reduction of group B streptococcal maternal and neonatal infections in preterm pregnancies with premature rupture of membranes through a rapid identification test. Am J Obstet Gynecol 1987;157:13-6.

29. Morales WJ, Lim DV, Walsh AF. Prevention of neonatal group B streptococcal sepsis by the use of a rapid screening test and selective intrapartum chemoprophylaxis. Am J Obstet Gynecol 1986;155:979-83.

30. Matorras R, Garcia-Perea A, Omenaca F, et al. Intrapartum chemoprophylaxis of early-onset group B streptococcal disease. Eur J Obstet Gynecol Reprod Biol 1991;40:57-62.

31. Tuppurainen N, Hallman M. Prevention of neonatal group B streptococcal disease: intrapartum detection and chemoprophylaxis of heavily colonized parturients. Obstet Gynecol 1989;73:583-7.

32. Allen U, Navas L, King S. Effectiveness of intrapartum penicillin prophylaxis in preventing early-onset group B streptococcal infection: results of a meta-analysis. Can Med Assoc J 1993;149:1659-65.

33. Minkoff H, Mead P. An obstetric approach to the prevention of early-onset group B $\beta$-hemolytic streptococcal sepsis. Am J Obstet Gynecol 1986;154:973-7.

34. Bray RE, Boe RW, Johnson WL. Transfer of ampicillin

Infectious Diseases and Immunization Committee Members: FBoucher, Centre hospitalier de I'Université Laval, Sainte-Foy, Québec;

DK Clogg (director responsible), Calgary, Alberta; $G$ Delage, Service de transfusion de sang de la Croix-Rouge canadienne, Montréal, Québec; E Ford-Jones, Hospital for Sick Children, Toronto, Ontario; T Jadajvi, Alberta Children's Hospital, Calgary, Alberta; S King, Hospital for Sick Children, Toronto, Ontario; N MacDonald (chair), Children's Hospital of Eastern Ontario, OHawa, Ontario;

D Speert, Research Centre, Vancouver, British Columbia. Consultants: U Allen, Children's Hospital of Eastern Ontario, Ottawa, Ontario (conultant on article only);

R Gold, The Hospital for Sick Children, Toronto, Ontario; SA Halperin, IWK Children's Hospital, Halifax, Nova Scotia;

BJ Law, Basic Medical Science Building, University of Manitoba. Winnipeg, Manitoba; V Marchessault, Children's Hospital of Eastern Ontario, Ottawa, Ontario. Liaisons: J Carlson, Disease Control Service.

Public Health Branch, Toronto, Ontario; L Pickering. American Academy of Pediatrics, Children's Hospital of the King's Daughters, Norfolk, Virginia; D Scheifele, Centre for Vaccine Evaluation, Division of Infectious Diseases, Vancouver, British Columbia; J Waters, Alberta Health, Communicable Disease Control, Edmonton, Alberta into fetus and amniotic fluid from maternal plasma in late pregnancy. Am J Obstet Gynecol1966;96:933-42.

35. Granato PA, Petosa MT. Evaluation of a rapid screening test for detecting group B streptococci in pregnant women. J Clin Microbiol 1991;29:1536-8.

36. Edwards MS. Group B streptococcal infections. Pediatr Infect Dis J 1990;9:778-81.

37. Wald ER, Dashefsky B, Green M, et al. Rapid detection of group B streptococci directly from vaginal swabs. J Clin Microbiol 1987;25:573-4.

38. Kontnick CM, Edberg SC. Direct detection of group B streptococci from vaginal specimens compared with quantitative culture. J Clin Microbiol 1990;28:336-9.

39. Skoll MA, Mercer BM, Baselski V, et al. Evaluation of two rapid group B streptococcal antigen tests in labor and delivery patients. Obstet Gynecol 1991;77:322-6.

40. Larsen JW, Dooley SL. Group B streptococcal infections: an obstetrical viewpoint. Pediatrics 1993;91:148-9.

41. Teres FO, Mattoras R, Perea AG, et al. Prevention of neonatal group B streptococcal sepsis. Pediatr Infect Dis J 1987;6:874.

42. Pylipow M, Gaddis M, Kinney JS. Selective intrapartum prophylaxis for group B streptococcus colonization: management and outcome of newborns. Pediatrics 1994;93:631-5.

43. Mohle-Boetani JC, Schuchat A, Plikaytis BD, et al. Comparison of prevention strategies for neonatal group $\mathrm{B}$ streptococcal infection: a population-based economic analysis. JAMA 1993;270:1442-8.

44. Strickland DM, Yeomans ER, Hankins GDV. Cost-effectiveness of intrapartum screening and treatment for maternal group B streptococci colonization. Am J Obstet Gynecol 1990;163:4-8.

45. Rouse DJ, Goldenberg RL, Cliver SP, et al. Strategies for the prevention of early-onset neonatal group B streptococcal sepsis: a decision analysis. Obstet Gynecol 1994;83:483-94.

46. Gibbs RS, Hall RT, Yow MD, et al. Consensus: Perinatal prophylaxis for group B streptococcal infection. Pediatr Infect Dis J 1992;11:179-83.

Fetus and Newborn Committee

Members: W Andrews, Janeway Child Health Centre, St John's, Newfoundland; D McMillan (chair), Foothills Hospital, Calgary, Alberta: T Perreault, Hôpital de Montréal pour enfants, Montréal, Québec; CR Walker, Children's Hospital of Eastern Ontario, Ottawa, Ontario; JL Watts (director responsible), Chedoke-McMaster Hospitals, Hamilton, Ontario. Consultants: A Allen, Grace Maternity Hospital, Halifax, Nova Scotia; E Outerbridge, Montreal Children's Hospital, Montreal, Quebec; S Saigal, McMaster University Medical Centre, Hamilton, Ontario.

Liaisons: R Natale, St Joseph's Health Centre, London, Ontario; W Oh, Women and Infants Hospital of Rhode Island, Providence, Rhode Island; A Papageorgiou, Jewish General Hospital, Montreal, Quebec; J Pinelli, Neonatal Nurses, Oakville, Ontario Maternal/Fetal Medicine Committee (The Society of Obstetricians and Gynaecologists of Canada)

Members: G Carson, Regina General Hospital, Regina, Saskatchewan: D Farquharson, Salvation Army Grace Hospital, Vancouver, British Columbia: A Lalonde, Society of Obstetricians and Gynaecologists of Canada (executive vicepresident); R Liston, The Women's Clinic, Halifax, Nova Scotia;

G Marquette, Hôpital Ste-Justine, Montréal, Québec;

P Mohide, Chedoke McMaster Hospital, Hamilton, Ontario; R Natale (chair), St Joseph's Health Centre, London, Ontario 


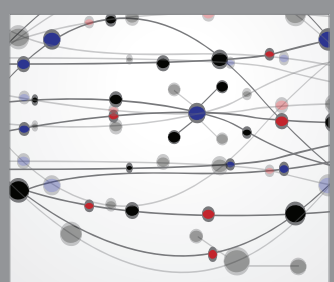

The Scientific World Journal
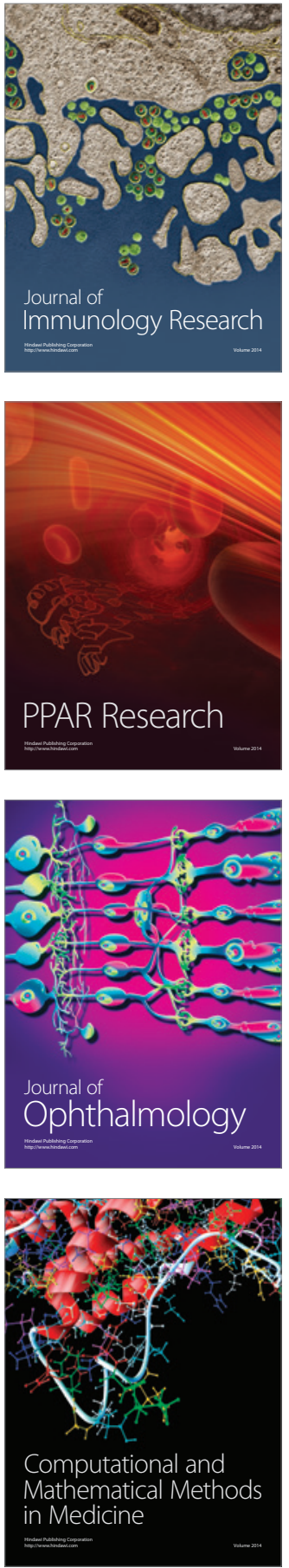

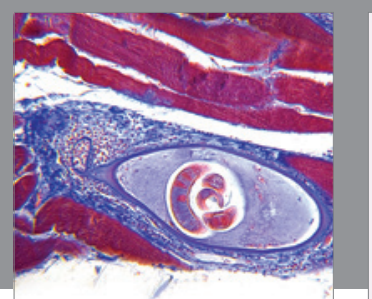

Gastroenterology Research and Practice

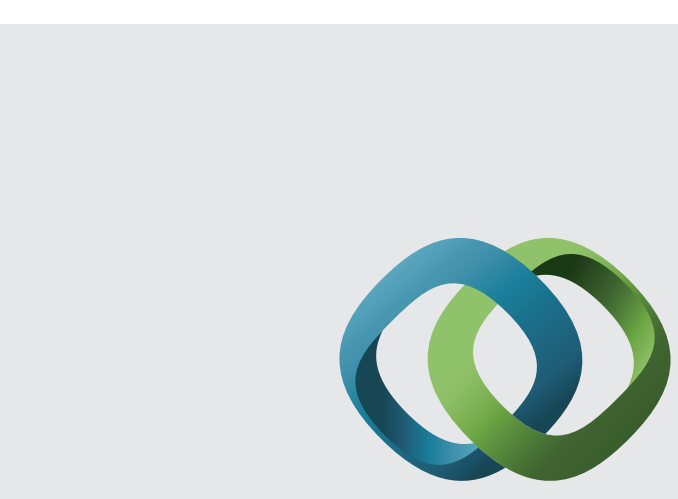

\section{Hindawi}

Submit your manuscripts at

http://www.hindawi.com
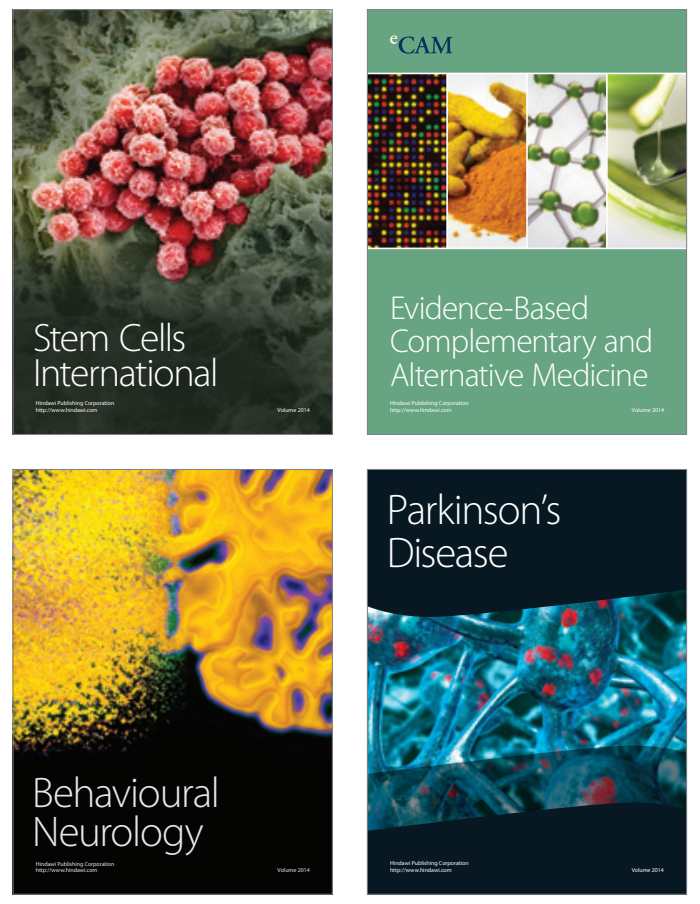
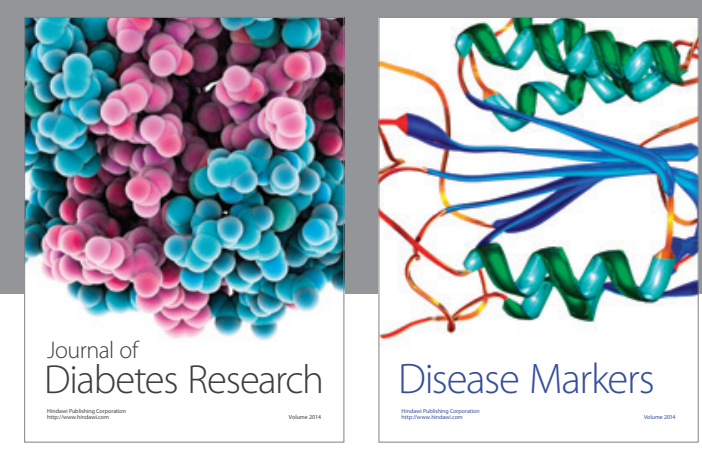

Disease Markers
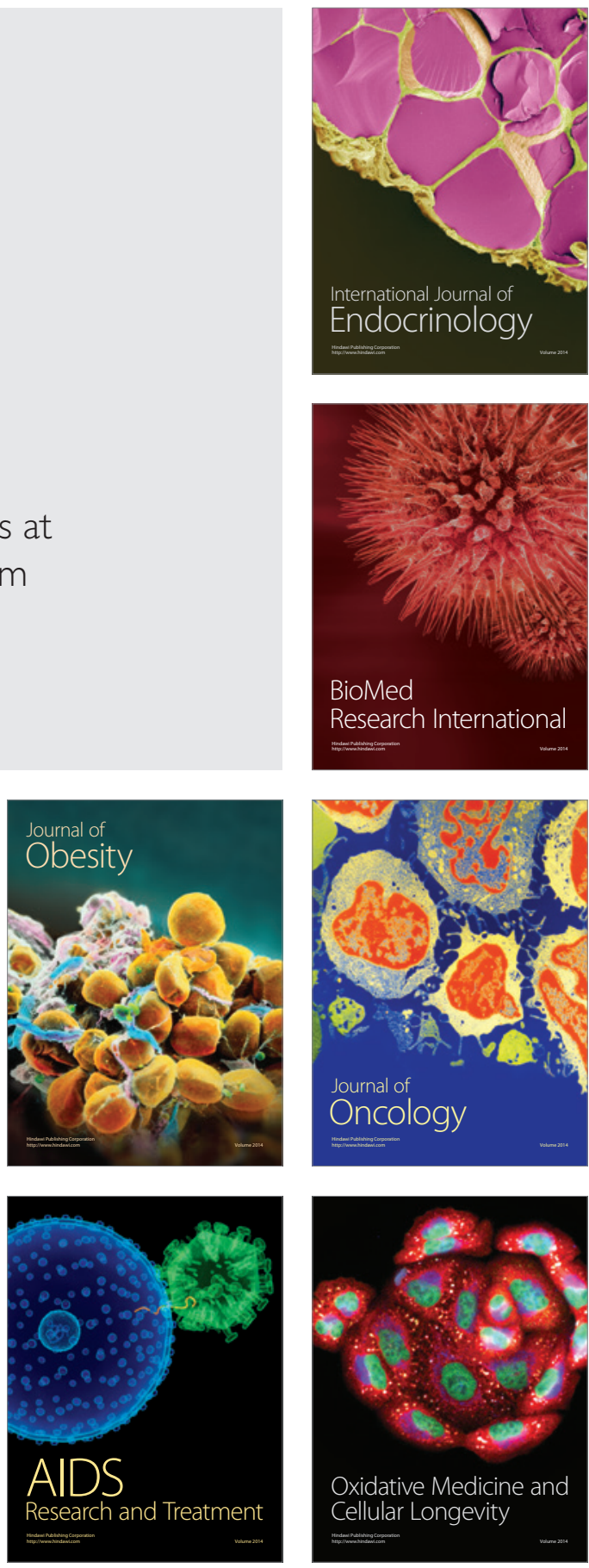